\title{
Effects of mechanical ventilation on diaphragm function and biology
}

\author{
G. Gayan-Ramirez, M. Decramer
}

Effects of mechanical ventilation on diaphragm function and biology. G. GayanRamirez, M. Decramer. (C) ERS Journals Ltd 2002.

ABSTRACT: The pathophysiological mechanisms of weaning from mechanical ventilation are not fully known, but there is accumulating evidence that mechanical ventilation induces inspiratory muscle dysfunction.

Recently, several animal models have provided potential mechanisms for mechanical ventilation-induced effects on muscle function. In patients, weaning difficulties are associated with inspiratory muscle weakness and reduced endurance capacity. Animal studies demonstrated that diaphragm force was already decreased after $12 \mathrm{~h}$ of controlled mechanical ventilation and this worsened with time spent on the ventilator.

Diaphragmatic myofibril damage observed after 3-days controlled mechanical ventilation was inversely correlated with maximal diaphragmatic force. Downregulation of the diaphragm insulin-like growth factor-I and MyoD/myogenin messenger ribonucleic acid occurred after $24 \mathrm{~h}$ and diaphragmatic oxidative stress and increased protease activity after $18 \mathrm{~h}$. In keeping with these findings, diaphragm fibre atrophy was shown after $12 \mathrm{~h}$ and reduced diaphragm mass was reported after $48 \mathrm{~h}$ of controlled mechanical ventilation.

These animal studies show that early alterations in diaphragm function develop after short-term mechanical ventilation. These alterations may contribute to the difficulties in weaning from mechanical ventilation seen in patients. Strategies to preserve respiratory muscle mass and function during mechanical ventilation should be developed. These may include: adaptation of medication, training of the diaphragm, stabilisation of the catabolic state and pharmacotherapy.

Eur Respir J 2002; 20: 1579-1586.
Respiratory Muscle Research Unit and Respiratory Division, Laboratory of Pneumology, University Hospitals, Katholieke Universiteit Leuven, Leuven, Belgium.

Correspondence: M. Decramer, Respiratory Division, University Hospitals, Herestraat 49, B-3000 Leuven, Belgium.

Fax: 3216346803

E-mail: marc.decramer@uz.kuleuven. ac.be

Keywords: Diaphragm, growth factors, intensive care unit, mechanical ventilation, transcription factors, weaning

Received: July 122002

Accepted after revision: August 12002

G. Gayan-Ramirez is a postdoctoral fellow of the "Fonds voor Wetenschappelijk Onderzoek-Vlaanderen" (Belgium) (FWO).
Ventilatory support is an essential life-saving therapy for intensive care patients with respiratory failure. Indications for mechanical ventilation include respiratory failure, status asthmaticus, chronic obstructive pulmonary disease (COPD), neuromuscular diseases, drug overdoses, and recovery from general anaesthesia. Most patients under mechanical ventilation are extubated in $<3$ days, but $\sim 20 \%$ require prolonged support [1]. In these patients, weaning procedures account for $>40 \%$ of total ventilator time [2], $60 \%$ for COPD patients [2]. Approximately $1-5 \%$ of mechanically ventilated patients repeatedly fail attempts to be weaned from mechanical ventilation and may become chronically ventilator-dependent [3]. This proportion increases to as much as $31-56 \%$ in some long-term ventilator units $[3,4]$. Chronic ventilator dependence is a major medical problem but it is also an extremely uncomfortable state for a patient, carrying important social implications [3].

Weaning failure has been extensively studied in the clinical literature and, in particular, attempts have been made to determine predictors, protocols and specific weaning strategies. Although the pathophysiological mechanisms of weaning from mechanical ventilation still remain to be determined, several factors are likely to contribute to weaning failure. These factors include inadequate ventilatory drive, respiratory muscle weakness, respiratory muscle fatigue, increased work of breathing or cardiac failure $[5,6]$. There is accumulating evidence that weaning problems are associated with failure of the respiratory muscles to resume ventilation. Conversely, although mechanical ventilation has been shown to protect the diaphragm against sarcolemma injury induced by sepsis [7], there is growing awareness of the amount of harm associated with invasive mechanical ventilation. In particular, mechanical ventilation seems to induce inspiratory muscle dysfunction. The nature and the cause of this inspiratory muscle dysfunction remain, however, poorly understood. In recent years, this has received renewed attention from different groups.

The aim of the present review is to describe the effects of mechanical ventilation on respiratory muscle function in mechanically ventilated patients and to compare these data with those obtained in animal models of mechanical ventilation. In particular, the recently described animal models will be put into perspective, as these data may offer new insights into 
the mechanisms of mechanical ventilation-induced effects on respiratory muscle function.

\section{Patient studies}

Muscle weakness, specifically impairment of respiratory muscles, is a well-known problem in the intensive care unit. Generally, muscle weakness is the consequence of the patient's illness. However, while respiratory rest during mechanical ventilation is suitable to reverse respiratory muscle fatigue, it may occur as a complicating factor during mechanical ventilation, since it may further exacerbate muscle weakness. However, in patients it remains difficult to demonstrate the effects of mechanical ventilation on the diaphragm directly. Nevertheless, weaning from mechanical ventilation presents a problem in $20-30 \%$ of the patients after prolonged mechanical ventilation [1], and even in 35-67\% of COPD patients [5]. Several factors are probably implicated but there is accumulating evidence that weaning problems are associated with the inability of the respiratory muscles to resume ventilation. Hence, the measurement of respiratory muscle function in mechanically ventilated patients appears a necessity, with the knowledge that the performance of respiratory muscles determines the ability to resume spontaneous ventilation following a period of mechanical ventilation. The tests used to assess respiratory muscle function in the intensive care unit have been recently reviewed [8] and will therefore not be described in the present review.

Respiratory muscle function in mechanically ventilated patients is essentially evaluated at the time of the weaning process. Imbalance between inspiratory load and respiratory muscle performance seems to play a major role in weaning failure.

Evidence of muscle fatigue was reported in patients recovering from acute respiratory failure [5, 9-12]. Thus, power spectrums of diaphragm electromyographic activity showed a reduction of the high-to-low frequency ratio in patients exhibiting difficulties during discontinuation of artificial ventilation. This was followed by respiratory alternans, paradoxical activation of abdominal muscles and a late reduction of both minute ventilation and respiratory rate with progressive respiratory acidaemia [9]. Similarly, a decrease in electromyographic activity of the diaphragm was also observed during controlled mechanical ventilation in patients recovering from respiratory failure in whom previous attempts at weaning had failed [11].

Similarly, in COPD patients ventilated due to respiratory failure, the ratio of transdiaphragmatic pressure $(P \mathrm{di}) / P$ di,max was reported to be higher in the group that failed to be weaned $[10,13,14]$. Diaphragmatic dysfunction was also suggested as gastric pressure was negative and associated by abdominal paradoxic motion [10]. In another study performed in mechanically ventilated patients with acute respiratory failure, it was shown that almost all the patients were breathing against a high inspiratory load that could lead to inspiratory muscle fatigue [12] (fig. 1).

In addition, when the load exceeded the capacity of

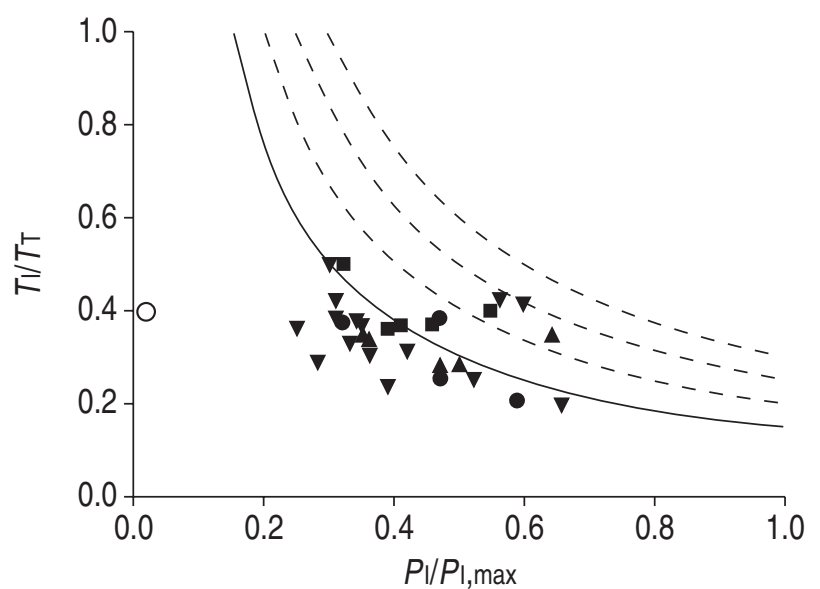

Fig. 1.- Inspiratory to total cycle duration ratio $\left(T_{\mathrm{I}} / T_{\mathrm{T}}\right)$ at the end of a spontaneous breathing trial versus inspiratory pressure expressed as a fraction of maximum $\left(P_{\mathrm{I}} / P_{\mathrm{I}}, \max \right)$ in chronic obstructive pulmonary disease $(\boldsymbol{O})$, acute respiratory distress syndrome $(\boldsymbol{\square})$, other pulmonary diseases $(\boldsymbol{\nabla})$ and acute respiratory failure of extrapulmonary origin $(\boldsymbol{\Delta})$. $\bigcirc$ : average value of ten normal subjects breathing with a minute volume similar to the mean minute volume of the patients. Reproduced with permission [12].

the respiratory muscles, slowing of the diaphragm maximum relaxation rate was reported in mechanically ventilated patients [15]. Thus, weaning difficulties seem to be associated with inspiratory muscle weakness and reduced endurance capacity $[12,15]$.

In neurological patients, maximal inspiratory mouth pressure (MIP) and maximal expiratory mouth pressure were reported to be lower in patients who failed to be weaned from mechanical ventilation [16]. This was also the case for MIP in elderly patients requiring reinstitution of ventilatory support [17].

Recently, PURro et al. [18] attempted to investigate the pathophysiological mechanisms underlying the inability to sustain spontaneous ventilation in ventilatordependent patients. They studied 39 patients, of which 28 had COPD, receiving mechanical ventilation through a tracheotomy, for a minimum of 3 weeks. They showed that in the patients who failed to be weaned from the ventilator, all indices of inspiratory muscle strength were lower compared to the weaned COPD patients or to the stable tracheotomised COPD patients. These included MIP and $P$ di,max. Both $P$ di $/$ $P$ di,max and intrapleural pressure $(P \mathrm{pl}) / P$ pl,max ratios were $>0.4$. In addition, the majority of the ventilatordependent patients failing the weaning process reached a tension-time index value close or above the 0.15 threshold. In the ventilator-dependent patients, the load/capacity balance was greater than in the weaned or stable patients.

Finally, the combination of mechanical ventilation with muscle-relaxing agents and/or high-dose steroids was reported to be associated with significant myopathy [19-23, 24]. Muscle weakness varied from mildto-severe. Muscle biopsy showed generalised fibre atrophy, myofibril necrosis and disorganisation with loss of thick myosin filaments and fibre vacuolation. The extent to which the diaphragm of these patients is also affected is not directly demonstrated but difficulty to wean from the ventilator is obvious in these 
patients. Several hypotheses have been proposed as potential mechanisms for these combined effects. Due to the fact that neuromuscular blockade agents are chemically close to corticosteroids, they may act in an additive manner with corticosteroids to induce myopathy [19]. In addition, increased sensitivity of the muscle to steroids may occur after denervation caused by neuromuscular blockade agents. Furthermore, some agents (corticosteroids, aminoglycosides antibiotics, lithium, magnesium sulphate, tetracyclines) or disorders (fluid-electrolytes-pH disturbances, renal failure, hepatic failure) may predispose to the myotoxic effect triggered by neuromuscular blockade agents [24].

\section{Animal studies}

The effects of mechanical ventilation on muscle function in animal models have been well studied and interest in these models has been recently revived. However, caution should be taken when interpreting the results of these studies because appropriate control groups (anaesthetised and spontaneously breathing animals) were not always included [25-27] and mechanical ventilation was combined with the administration of antibiotics and neuromuscularblocking agents [28], which are also known to compromise diaphragmatic function [29].

\section{Diaphragm strength, morphological and metabolic properties}

The first animal model of mechanical ventilation examined the effects of 48-h mechanical ventilation on the in vitro contractile properties of the diaphragm and of two peripheral muscles [25]. This study showed that, compared to unanaesthetised rats, diaphragmatic force was reduced after mechanical ventilation, while the force of the soleus (a slow muscle) was increased at low stimulation frequencies and the force of the extensor digitorum longus (a fast muscle) remained unchanged. The mass of the three muscles was significantly reduced after mechanical ventilation. Interestingly, crural diaphragm and soleus mass were mostly affected, with a decrease of $\sim 20 \%$, while the mass of the costal diaphragm and the extensor digitorum longus were reduced by 14 and $11 \%$, respectively. Finally, muscle total protein content, citrate synthase (an enzyme marker of oxidative capacity) and lactate dehydrogenase (a glycolytic marker enzyme) activities were not affected in any of the muscles studied. Appropriate controls were not used in this study, therefore, part of the effects seen after mechanical ventilation may have been due to the effects of anaesthesia or undernutrition.

In another study, mechanical ventilation, prolonged to 3-7 days in combination with antibiotics and paralysing agents, led to diaphragm weight loss when compared to anaesthetised and spontaneously breathing rats [28]. There was also an increase in diaphragm fibres co-expressing myosin heavy chain (MHC)-I and -II at the expense of the MHC-II population.
However, caution should be taken since only two or three animals per group were examined in this study and the antibiotics and paralysing agents may have contributed to the diaphragmatic changes seen after mechanical ventilation.

Similarly in piglets, 5 days of mechanical ventilation without paralysis or antibiotics resulted in a fall of $P$ di at all frequencies $(\sim 20 \%)$ compared to baseline measurements [27]. This was accompanied by a $30 \%$ decrease in evoked compound muscle action potential (CMAP) amplitude. Finally, CMAP threshold and latency did not change over the course of the 5 days' mechanical ventilation, suggesting that nerve function was intact. Likewise, repetitive stimulation at $3 \mathrm{~Hz}$ did not result in significant diminution in amplitude over time, indicating that neuromuscular junction transmission was intact.

A longer duration of mechanical ventilation (11 days) in baboons resulted in a $25 \%$ decrease in $P$ di,max and a 36\% decrease in diaphragmatic endurance capacity [26]. There were no changes in haemodynamics, lung volume or oxygenation but pulmonary histopathology showed localised alveolar filling in two animals and mild focal pneumonitis in the remaining animals. However, the sample size of this study was very low ( $n=3$ for phrenic stimulation), and diaphragm mass was not measured and contractile properties were not studied. In addition, both antibiotics and paralysing agents were used in combination with mechanical ventilation and they may have played a part in the decreased diaphragmatic strength observed. The effect of anaesthesia was also not distinguished from the effects of mechanical ventilation.

Recently, several studies have been performed to examine the time course of mechanical ventilationinduced alterations on diaphragm function [30, 31]. A decrease in in vitro maximal specific force was reported in the rat diaphragm soon after $12 \mathrm{~h}$ of controlled mechanical ventilation [30] (fig. 2). In addition, this decrease increased with time spent on the ventilator $(-18 \%$ and $-46 \%$ after 12 and $24 \mathrm{~h}$ of mechanical ventilation, respectively). There was also an inverse relationship between the mechanical ventilation duration and the values of maximal specific force $(\mathrm{r}=0.88)$.

Likewise, SASSOON et al. [31] looking at the time course of the in vivo and in vitro diaphragmatic force alterations following 1 or 3 days of controlled mechanical ventilation, reported a time-dependent decline in rabbit diaphragmatic function. In particular, $P$ di,max was reduced by 27 and $51 \%$ after 1 and 3 days of controlled mechanical ventilation, respectively, while this pressure remained unchanged in anaesthetised animals with continuous positive airway pressure (CPAP). Pdi was actually significantly decreased at all stimulation frequencies after 3 days of mechanical ventilation compared to animals with 3 days of CPAP. Interestingly, in vitro measurements in the same animals showed that diaphragm force generating capacity significantly declined after 3 days of controlled mechanical ventilation but not after $24 \mathrm{~h}$. This is in contrast to the findings of Powers et al. [30] and to the present authors' data. Indeed, the present authors observed a significant decrease in 


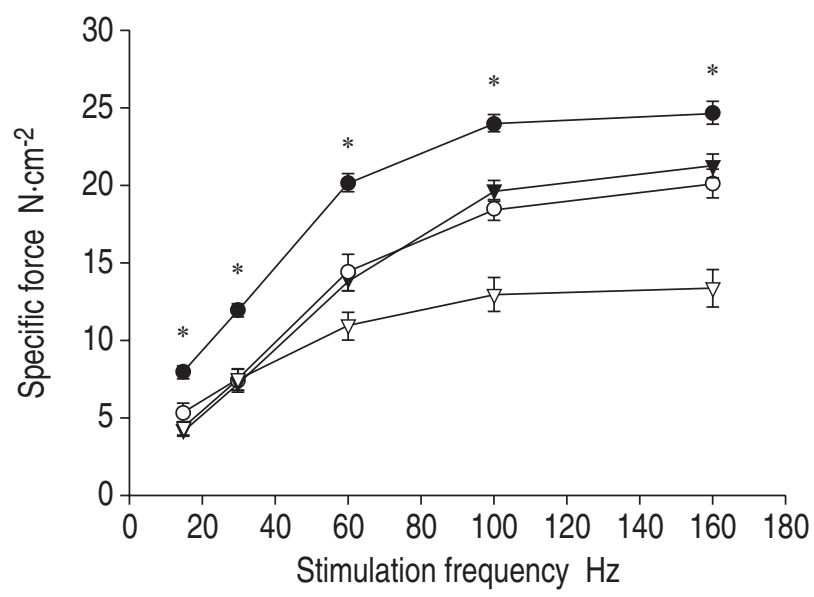

Fig. 2.-In vitro diaphragm force/frequency curve in the diaphragm of controls $(\bigcirc)$ and ventilated rats $(\bigcirc: 12 \mathrm{~h} ; \boldsymbol{\nabla}: 18 \mathrm{~h} ; \nabla$ : $24 \mathrm{~h}$ ). Data are presented as mean \pm SEM. *: $\mathrm{p}<0.05$ versus control. Reproduced with permission [30].

in vitro diaphragmatic force in rats after 24-h controlled mechanical ventilation and this decrease was more pronounced in mechanically ventilated rats than in the anaesthetised and spontaneously breathing rats using the same instrumentation [32]. There are also discrepancies concerning the effects of mechanical ventilation on diaphragm fibre dimensions. Indeed, while the group of SHANELY et al. [33] reported a reduction in the cross-sectional area of all muscle fibre types in rats after 18-h mechanical ventilation, no changes in fibre dimensions were observed by SASSOON et al. [31] in the rabbit diaphragm either after 1 or 3 day mechanical ventilation. In the latter study, proportions of fibres expressing the different MHC isoforms and their relative contribution to total crosssectional area of the diaphragm were unchanged as was succinic dehydrogenase enzyme activity. It is not known whether species differences contribute to the discrepancies between these two studies but it should be noted that the fibre composition of rat and rabbit diaphragms differs, as rat diaphragm contains predominantly MHC-2X and rabbit diaphragm contains a similar proportion of $\mathrm{MHC}$-slow and $\mathrm{MHC}-2 \mathrm{~A}$ and almost no MHC-2X.

\section{Ultrastructural changes}

Ultrastructural data have showed that myofibril damage was present in the rabbit diaphragm (fig. 3) and not in the soleus after 3 days of controlled mechanical ventilation [31]. In addition, an inverse relationship was present between maximal in vitro diaphragmatic force and the volume of abnormal myofibrils $(\mathrm{r}=0.82, \mathrm{p}<0.01)$, such that diaphragm injury explained $66 \%$ of the variance in the reduction of tetanic force [31].

\section{Molecular level}

At the biological level, $18 \mathrm{~h}$ of controlled mechanical ventilation was shown to increase diaphragmatic protein degradation along with elevated calpain and proteosome activity [33]. This was associated with an increase in lipid peroxidation and protein oxidation as both 8-isoprostane and protein carbonyls were increased after mechanical ventilation.

Furthermore, adaptations of the diaphragm at the molecular level were also observed after $24 \mathrm{~h}$ of controlled mechanical ventilation. Thus, the insulin-like growth factor (IGF)-I messenger ribonucleic acid (mRNA) levels were significantly reduced in the
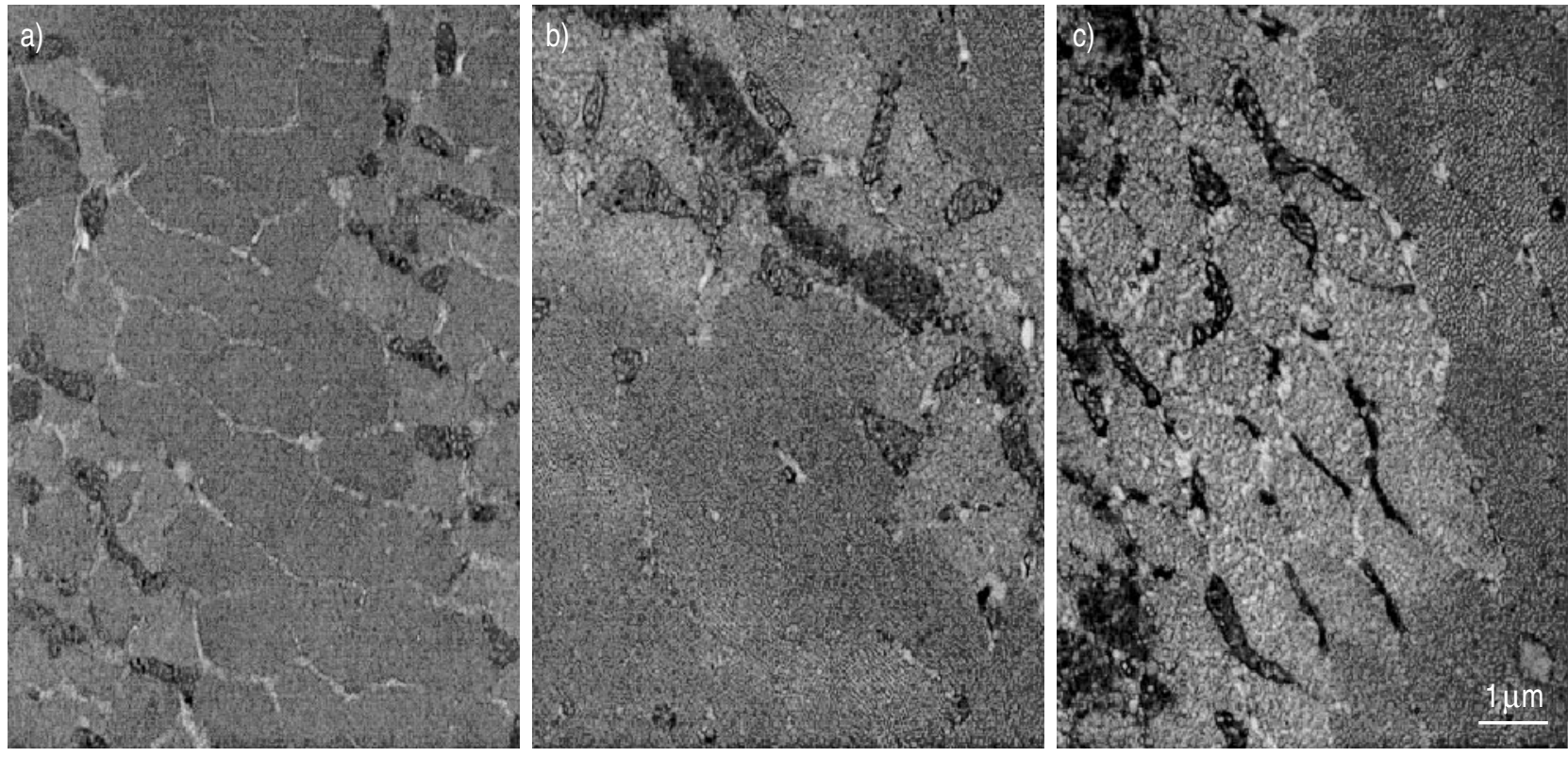

Fig. 3.-Electron-microscopic cross-sections of diaphragm myofibrils. a) Control, b) 3 days of continuous positive airway pressure and c) 3 days of controlled mechanical ventilation. Reproduced with permission [31]. 


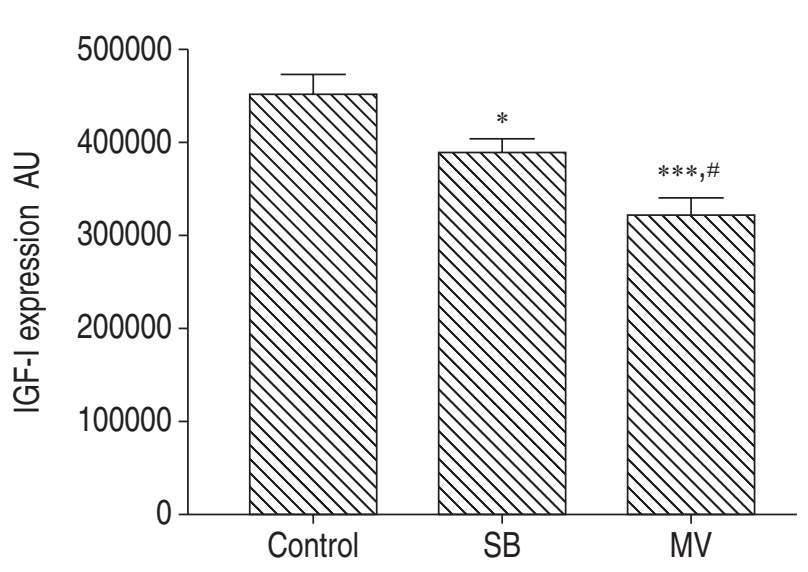

Fig. 4.-Insulin-like growth factor (IGF)-I messenger ribonucleic acid levels determined by reverse transcriptase polymerase chain reaction in the diaphragm of control rats, spontaneously breathing rats (SB) and rats under mechanical ventilation (MV). Band intensities of the IGF-I amplified fragment were normalised to the corresponding L32 amplification signals (house-keeping gene). Data are presented as means \pm SD. *: $\mathrm{p}<0.05$; and $* * *: \mathrm{p}<0.001$ versus control; ${ }^{\#}: \mathrm{p}<0.05$ versus $\mathrm{SB}$

diaphragm of anaesthetised spontaneously breathing rats $(-14 \%)$ but this downregulation was more pronounced in mechanically ventilated rats $(-29 \%)$ [32] (fig. 4). There was also a significant positive correlation between the diaphragm IGF-I mRNA expression and its in vitro force production. In addition, the $\mathrm{MyoD} / \mathrm{myogenin}$ ratio, which may reflect ongoing changes in isoform switch, was decreased in the diaphragm after $24-\mathrm{h}$ mechanical ventilation, as were the transcript levels of the fast isoforms of both MHC and sarcoplasmic-endoplasmic reticulum calcium adenosine trisphosphatase (SERCA) pump [34, 35].

\section{Potential mechanisms of mechanical ventilation- induced effects on diaphragm function}

Independently of anaesthesia, the detrimental effect of mechanical ventilation is likely to be multifactorial. First, during mechanical ventilation, electrical activity to the diaphragm is absent. This alone affects the transcription of muscle regulatory factors [36, 37], with all of its consequences, i.e. muscle phenotype shift [38-40]. Secondly, the rhythmical passive shortening of the diaphragm may also have a negative effect on the muscle, as it may cause either fibre injury and/or muscle phenotype adaptation. Thirdly, as mechanical ventilation is utilised to unload the diaphragm, it causes muscle deconditioning per se.

Data in the literature show that the effects of diaphragm denervation depend on whether respiratory centre-phrenic motoneurone transmission is intact and mainly on neutrotrophic influence [41]. Thus, diaphragm fibre adaptation was greater in the spinal cord hemisection (C2) model than after unilateral denervation of the diaphragm or tetrodotoxin nerve blockade [41]. However, in the case of mechanical ventilation, true denervation is not present in the sense that both phrenic nerves remain intact, nerve conduction and neuromuscular transmission being unaffected [27]. Thus, factors other than denervation probably contribute to the effects of mechanical ventilation on diaphragm function to a greater extent.

The effects of skeletal muscle deconditioning or unloading are well known, but all the data pertain to hindlimb unloading models where a slow-to-fast phenotype transition regarding $\mathrm{MHC}$ or SERCA isoforms has been described after 7-10 days [42, 43]. In mechanical ventilation, however, the diaphragm is unloaded in a specific way, as it continuously undergoes repetitive shortening cycles, during which the sarcomere length really shortens. Therefore, the current authors examined the effects of immobilisation alone or combined with a similar range of passive shortening on the gastrocnemius muscle, in order to unravel which of the consequences of mechanical ventilation, passive shortening or muscle unloading (deconditioning), would be responsible for the effects observed in the diaphragm after mechanical ventilation [44]. It was found that muscle deconditioning rather than passive shortening was likely to be responsible for impaired muscle function after mechanical ventilation, as passive shortening did not produce any additional effects on the expression levels of the studied factors compared to deconditioning. Hence, the nature of the effect of gastrocnemius deconditioning was similar to that seen in the diaphragm after mechanical ventilation, although less pronounced in magnitude [44].

Impairment in excitation-contraction coupling may also occur in the diaphragm during mechanical ventilation. Indeed, any factor capable of reducing calcium release from the sarcoplasmic reticulum and/ or calcium sensitivity would alter force production. Reduction in CPAP amplitude and fall in force output, as reported in the diaphragm of a piglet after 3 days of mechanical ventilation, suggest that either excitation-contraction coupling or membrane depolarisation may be involved in the diaphragm dysfunction [27]. Along the same lines, the current authors' data show that the expression of the fast SERCA pump isoform was reduced in the mechanically ventilated diaphragm after only $24 \mathrm{~h}$ [35]. The effects of mechanical ventilation on the diaphragm resting membrane action potential still remain to be determined.

Intrinsic alterations in diaphragm myofibrils are also involved in the detrimental effects of mechanical ventilation on the diaphragm. Indeed, as described previously, 18-h mechanical ventilation in rats resulted in a generalised diaphragm fibre atrophy [30], while myofibril damage was observed in the rabbit diaphragm after 3 days of controlled mechanical ventilation [31]. Finally, it was suggested that mechanical ventilation promoted diaphragmatic oxidative stress and increased protease activity, protein degradation, and calpain and proteosome activity. Protein carbonyls and 8-isoprostane have also been found to be increased in the rat diaphragm as soon as $18 \mathrm{~h}$ after mechanical ventilation [33]. 


\section{Comments}

All the animal studies discussed in this review have examined the effect of mechanical ventilation on diaphragm function using controlled mechanical ventilation, although this mode of ventilation is not the mode commonly used in clinical practice. However, patients with acute respiratory distress remain ventilated through sedation with or without paralysis.

These animal studies are of particular interest as they show that mechanical ventilation itself exerts deleterious effects on diaphragm function independently of anaesthesia. These effects indicate that only $12 \mathrm{~h}$ of controlled mechanical ventilation can result in impaired diaphragm function, the magnitude and nature of these effects worsening with the time spent on the ventilator.

These data are of conceptual interest for clinical medicine as they clearly show that early alterations in diaphragm function develop after short-term mechanical ventilation and may contribute to the difficulties in weaning from mechanical ventilation observed in patients. In particular, it should be noted that all these studies were performed on animals with normal diaphragm function in whom $24 \mathrm{~h}$ of mechanical ventilation already induced pronounced alterations in their normal diaphragm. If mechanical ventilation would be applied to animals with already impaired diaphragm function, it would probably result in more severe changes in diaphragm intrinsic properties. These findings should be taken into account when mechanical ventilation is used in patients with respiratory muscle weakness, as it may worsen their diaphragm function and lead to weaning failure. These studies underline that even short-term mechanical ventilation may be a problem for these patients in whom prolongation of mechanical ventilation would be expected to be necessary with more risk for further development of diaphragm weakness.

\section{Perspectives}

There are still several questions that need to be addressed in order to develop preventive or curative therapeutic strategies.

In particular, it remains to be established whether the combination of mechanical ventilation with drugs, such as corticosteroids, paralysing agents and aminoglycoside antibiotics, promotes muscle weakness more than mechanical ventilation alone. Preliminary data from the current authors' group suggest this is the case for the combination mechanical ventilation and high-dose corticosteroids [45]. Therefore, attempts should be directed at minimising the dosage of the paralysing agents and/or the combination with corticosteroids and eventually the use of nonsteroidal neuromuscular-blocking agents may be convenient.

Similarly, because all the critically ill patients are catabolic, a state known to affect muscle function, the deleterious effect of catabolism on muscle function is likely to be exacerbated when combined with inactivity (bed rest, mechanical ventilation). This may lead to disuse atrophy, also in the diaphragm. An attractive alternative to prevent human diaphragm atrophy caused by prolonged inactivation would to be to apply brief periods of daily phrenic nerve stimulation, as this has been shown to be efficient in patients with high spinal cord injury [46]. Although it may be energy consuming, an adapted and early pulmonary rehabilitation programme may be applied, as it has also been shown to be beneficial [47]. Likewise, inspiratory muscle-resistive training may improve respiratory muscle strength and endurance and help the patients to be weaned from the ventilator [48].

Careful nutritional treatment aimed at increasing mass and improving function of the respiratory muscles may help to minimise inspiratory muscle fatigue and thus to reduce mechanical ventilation duration. This process is, however, difficult and time consuming.

Finally, the use of pharmacotherapy may be a valuable tool. Anabolic agents and/or treatment with growth factors such as IGF-I and growth hormone may help to minimise muscle atrophy. Growth hormone treatment has, indeed, been shown to improve inspiratory force of mechanically ventilated patients [49]. However, treatment duration and dose are probably important factors as low-dose and shortterm treatment with growth hormone neither improved peripheral muscle strength nor the duration of ventilatory support although it did promote nitrogen retention [50].

All these therapeutic strategies need further experiments to confirm their potential efficiency. For these purposes, animal models may be helpful, keeping in mind that extrapolation of these animal model data to patients should be performed with caution.

\section{References}

1. Esteban A, Frutos F, Tobin MJ, et al. A comparison of four methods of weaning patients from mechanical ventilation. $N$ Engl J Med 1995; 332: 345-350.

2. Esteban A, Alia I, Ibanez J, Benito S, Tobin MJ. Modes of mechanical ventilation and weaning. A national survey of Spanish hospitals. The Spanish Lung Failure Collaborative Group. Chest 1994; 106: 1188-1193.

3. Celli B. Home mechanical ventilation. In: Tobin MJ, ed. Principles and Practice of Mechanical Ventilation. New York, McGraw Hill Inc., 1994; pp. 619-629.

4. Scheinhorn D, Chao DC, Stearn-Hassenpflug M. Post-ICU mechanical ventilation. Treatment of 1,123 patients at a regional weaning center. Chest 1997; 111: 1654-1659.

5. Sporn PH, Morganroth M. Discontinuation from mechanical ventilation. Clin Chest Dis 1988; 9: 113126.

6. Epstein SK. Weaning from mechanical ventilation. Respir Care 2002; 47: 454-466.

7. Ebihara S, Hussain SNA, Danialou G, Cho WK, Gottfried SB, Petrof BJ. Mechanical ventilation protects against diaphragm injury in sepsis. $\mathrm{Am}$ J Respir Crit Care Med 2002; 165: 221-228. 
8. Tobin MJ, Brochard L, Rossi A. Assessment of respiratory muscle function in the intensive care unit. Am J Respir Crit Care Med 2002; 166: 610-623.

9. Cohen CA, Zagelbaum G, Gross D, Roussos C, Macklem PT. Clinical manifestations of inspiratory muscle fatigue. Am J Med 1982; 73: 308-316.

10. Pourriat JL, Lamberto Ch, Hoang PH, Fournier JL, Vasseur B. Diaphragmatic fatigue and breathing pattern during weaning from mechanical ventilation in COPD patients. Chest 1986; 90: 703-707.

11. Brochard L, Harf A, Lorino H, Lemaire F. Inspiratory pressure support prevents diaphragmatic fatigue during weaning from mechanical ventilation. Am Rev Respir Dis 1989; 139: 513-521.

12. Zakynthinos SG, Vassilakopoulos T, Roussos C. The load of inspiratory muscles in patients needing mechanical ventilation. Am J Respir Crit Care Med 1995; 152: 1248-1255.

13. Vassilakopoulos T, Zakynthinos S, Roussos C. The tension-time index and the frequency/tidal volume ratio are the major pathophysiologic determinants of weaning failure and success. Am J Respir Crit Care Med 1998; 158: 378-385.

14. Appendini L, Purro A, Patessio A, et al. Partitioning of inspiratory muscle workload and pressure assistance in ventilator-dependent COPD patients. $\mathrm{Am}$ J Respir Crit Care Med 1996; 154: 1301-1309.

15. Goldstone JC, Green M, Moxham J. Maximum relaxation rate of the diaphragm during weaning from mechanical ventilation. Thorax 1994; 49: 54-60.

16. Vallverdu I, Calaf N, Subirana M, Net A, Benito S, Mancebo J. Clinical characteristics, respiratory functional parameters, and outcome of a two-hour T-piece trial in patients weaning from mechanical ventilation. Am J Respir Crit Care Med 1998; 158: 1588-1562.

17. Krieger BP, Ershowsky PF, Becker DA, Gazeroglu HB. Evaluation of conventional criteria for predicting successful weaning from mechanical ventilatory support in elderly patients. Crit Care Med 1989; 17: 585861.

18. Purro A, Appendini L, De Gaetano A, Gudjonsdottir M, Donner CF, Rossi A. Physiologic determinants of ventilator dependence in long-term mechanically ventilated patients. Am J Respir Crit Care Med 2000; 161: 1855-1862.

19. Kaplan PW, Rocha W, Sanders DB, D'Souza B, Spock A. Acute steroid-induced tetraplegia following status asthmaticus. Pediatrics 1986; 78: 121-123.

20. Danon MJ, Carpenter S. Myopathy with thick filament (myosin) loss following prolonged paralysis with vencuronium during steroid treatment. Muscle Nerve 1991; 14: 1131-1139.

21. Griffin D, Fairman N, Coursin D, Rawsthorne L, Grossman JE. Acute myopathy during treatment of status asthmaticus with corticosteroids and steroidal muscle relaxants. Chest 1992; 102: 510-514.

22. Hirano M, Ott BR, Raps EC, et al. Acute quadriplegic myopathy: a complication of treatment with steroids, nondepolarizing blocking agents, or both. Neurology 1992; 42: 2082-2087.

23. Lacomis D, Smith TW, Chad DA. Acute myopathy and neuropathy in status asthmaticus-case report and literature review. Muscle Nerve 1993; 16: 84-90.

24. Gooch JL. Prolonged paralysis after neuromuscular blockade. J Toxicol Clin Toxicol 1995; 33: 419-426.

25. Le Bourdelles G, Viires N, Boczkowski J, Seta N, Pavlovic D, Aubier M. Effects of mechanical ventilation on diaphragmatic contractile properties in rats. $\mathrm{Am}$ J Respir Crit Care Med 1994; 149: 1539-1544.

26. Anzueto A, Peters JI, Tobin MJ, et al. Effect of prolonged controlled mechanical ventilation on diaphragmatic function in healthy adult baboon. Crit Care Med 1997; 25: 1187-1190.

27. Radell PJ, Remahl S, Nichols DG, Eriksson LI. Effects of prolonged mechanical ventilation and inactivity on piglet diaphragm function. Intensive Care Med 2002; 28: 358-364.

28. Yang L, Luo J, Lin C, Gottfried SB, Petrof BJ. Effect of long-term mechanical ventilation on rat diaphragm mass and myosin heavy chain expression. Am J Respir Crit Care Med 1997; 155: A509.

29. Douglass JA, Tuxen DV, Horne M, et al. Myopathy in severe asthma. Am Rev Respir Dis 1992; 146: 517519.

30. Powers SK, Shaneley RA, Coombes JS, et al. Mechanical ventilation results in progressive contractile dysfunction in the diaphragm. J Appl Physiol 2002; 92: 1851-1858.

31. Sassoon CSH, Caiozzo VJ, Manka A, Sieck GC. Altered diaphragm contractile properties with controlled mechanical ventilation. J Appl Physiol 2002; 92: 2585-2595.

32. Gayan-Ramirez G, de Paepe K, Decramer M. Shortterm mechanical ventilation alters diaphragm contractile properties and insulin-like growth factor-I expression in rats. Am J Respir Crit Care Med 2001; 163: A802.

33. Shanely RA, Lennon SL, Yimlamai T, et al. Mechanical ventilation-induced diaphragmatic atrophy is associated with oxidative injury and increased proteolytic activity. Am J Respir Crit Care Med 2002; 165 : A261.

34. Racz G, Gayan-Ramirez G, de Paepe K, Zador E, Wuytack F, Decramer M. Short-term mechanical ventilation is associated with alterations in transcription factor and Id-protein mRNA in rat diaphragm. Eur Respir J 2001; 68: 420s.

35. Racz G, Gayan-Ramirez G, de Paepe K, Zador E, Wuytack F, Decramer M. Short-term mechanical ventilation alters myosin heavy chain and SERCA pump mRNA levels in rat diaphragm. Am $J$ Respir Crit Care Med 2002; 165: A260.

36. Carlsen H, Gundersen K. Helix-loop-helix transcription factors in electrically active and inactive skeletal muscles. Muscle Nerve 2000; 23: 1374-1380.

37. Eftimie R, Brenner HR, Buonanno A. Myogenin and MyoD join a family of skeletal muscle genes regulated by electrical activity. Proc Natl Acad Sci USA 1991; 88: 1349-1353.

38. Pette D. Fiber transformation and fiber replacement in chronically stimulated muscle. $J$ Heart Lung Transplant 1992; 11: S299-S305.

39. Gundersen K, Leberer E, Lomo T, Pette D, Staron RS. Fibre types, calcium-sequestering proteins and metabolic enzymes in denervated and chronically stimulated muscles of the rat. J Physiol 1988; 398: 177-189.

40. Hu P, Zhang K-M, Wright LD, Spratt JA, Briggs FN. Correlations between MyoD, myogenin, SERCA1, SERCA2 and phospholamban transcripts during transformation of type-II to type-I skeletal muscle fibers. Eur J Physiol 1997; 434: 209-211.

41. Zhan WZ, Miyata H, Prakash YS, Sieck GC. Metabolic and phenotypic adaptations of diaphragm 
muscle fibers with inactivation. J Appl Physiol 1997; 82: $1145-1153$.

42. Talmadge RJ. Myosin heavy chain isoform expression following reduced neuromuscular activity: potential regulatory mechanisms. Muscle Nerve 2000; 23: 661679.

43. Stevens L, Firinga C, Gohlsch B, Bastice B, Mounier Y, Pette D. Effects of unweighting and clenbuterol on myosin light and heavy chain in fast and slow muscles of rat. Am J Physiol Cell Biol 2000; 279: C1558-C1563.

44. Racz G, Gayan-Ramirez G, Zador E, Wuytack F, Decramer M. Short-term passive shortening and deconditioning alters mRNA levels of transcription factors in rat gastrocnemius. Am J Respir Crit Care Med 2002; 165: A261.

45. Gayan-Ramirez G, Derthoo D, van Kuyck K, Decramerk M. Corticosteroid treatment worsens the detrimental effect of mechanical ventilation on rat diaphragm function. Eur Respir J 2002; 20: 222S.
46. Ayas NT, McCool FD, Gore R, Lieberman SL, Brown R. Prevention of human diaphragm atrophy with short periods of electrical stimulation. Am J Respir Crit Care Med 1999; 159: 2018-2020.

47. Nava S. Rehabilitation of patients admitted to a respiratory intensive care unit. Arch Phys Med Rehabil 1998; 79: 849-854.

48. Aldrich TK, Karpel JP, Uhrlass RM, Sparapani MA, Eramo D, Ferranti R. Weaning from mechanical ventilation: adjunctive use of inspiratory muscle resistive training. Crit Care Med 1989; 17: 143-147.

49. Knox JB, Wilmore DW, Demling RH, Sarraf P, Santos AA. Use of growth hormone for postoperative respiratory failure. Am J Surg 1996; 171: 576-580.

50. Pichard C, Kyle U, Chevrolet J-C, et al. Lack of effects of recombinant growth hormone on muscle function in patients requiring prolonged mechanical ventilation: a prospective, randomized, controlled study. Crit Care Med 1996; 24: 403-413. 